\title{
INHOMOGENEOUS DIRICHLET BOUNDARY CONDITION IN THE A POSTERIORI ERROR CONTROL OF THE OBSTACLE PROBLEM
}

\author{
SHARAT GADDAM AND THIRUPATHI GUDI
}

\begin{abstract}
We propose a new and simpler residual based a posteriori error estimator for finite element approximation of the elliptic obstacle problem. The results in the article are two fold. Firstly, we address the influence of the inhomogeneous Dirichlet boundary condition in a posteriori error control of the elliptic obstacle problem. Secondly by rewriting the obstacle problem in an equivalent form, we derive simpler a posteriori error bounds which are free from $\min / \max$ functions. To accomplish this, we construct a post-processed solution $\tilde{u}_{h}$ of the discrete solution $u_{h}$ which satisfies the exact boundary conditions although the discrete solution $u_{h}$ may not satisfy. We propose two post processing methods and analyze them. We remark that the results known in the literature are either for the homogeneous Dirichlet boundary condition or that the estimator is only weakly reliable in the case of inhomogeneous Dirichlet boundary condition.
\end{abstract}

\section{INTRODUCTION}

The elliptic obstacle problem is one of the popular prototype models for the study of elliptic variational inequalities. The applications of variational inequalities are enormous in the modern scientific computing world, e.g. in contact mechanics, option pricing and fluid flow problems. The numerical analysis of these class of problems is an interesting subject as they offer challenges both in theory and computation. We refer to the books [3, 22, 35, 43] for the theory of variational inequalities and their corresponding numerical analysis. Apart from these, we refer to the articles [12, 20] and the recent articles [10, 11, 32, 47, 48] for the convergence analysis of finite element methods for the obstacle problem. The obstacle problem exhibits free boundaries where the regularity of the solution is affected. It is worth remarking here that the location of a free boundary is not known a priori. Adaptive finite element methods based on reliable and efficient a posteriori error estimates are of particular interest in this contest as they can capture the free boundaries by local mesh refinement around them. In designing any of adaptive schemes, the first step is to derive some computable error estimators which are both reliable and efficient, see [1] for error analysis of various type. There are many works in deriving residual based a posteriori error estimates for the obstacle problem, see [2, 5, 8, 16, 23, 24, 25, 26, 37, 39, 44, 50] and see [4, 27, 28, 49]. In recent years, much of research is focused on proving the convergence of adaptive methods based on a posteriori error estimates. In this direction, we refer to [13, 14, 21, 42, 40] for the

1991 Mathematics Subject Classification. 65N30, 65N15.

Key words and phrases. finite element, a posteriori error estimate, obstacle problem, variational inequalities, Lagrange multiplier, reliable, efficient. 
work related to the obstacle problem. Further, we refer [6, 19, 29, 33] and [41, 7, 30, 51] for the work related to the numerical approximation of the Signorini contact problem.

In many occasions, it is assumed for convenience in the a posteriori error analysis of obstacle problems that the Dirichlet data is either zero or trace of a finite element function. However it is not clear if the error estimator with homogeneous Dirichlet boundary condition is reliable and efficient in the energy norm up to some Dirichlet data oscillations. The answer to this question so far seems to be not clear as it can be seen in [21, Section 4.2] that the error estimator is proved only weakly reliable with nonhomogeneous Dirichlet boundary data oscillations. Exceptions to this question hold for the local error analysis (estimates in maximum norm) in [38, 37] where reliable and efficient error estimates were derived for general Dirichlet boundary data. One of the difficulties in the energy norm error estimate arises due to the fact that the error $u-u_{h}$ does not belong to $H_{0}^{1}(\Omega)$. It will be difficult to argue with the residual directly using the error $u-u_{h}$. We may think of introducing an auxiliary problem correcting this as in the case of linear elliptic problems, but with this the estimator will consists of the unknown solution of that auxiliary problem. In this article, we introduce two options of constructing a post-processed solution in which one of them can be computed explicitly. We also derive some estimates for the discrete solution and its post-processed solution. Thereby, we address the question of proving the reliability and efficiency of the error estimator up to some Dirichlet data oscillations as in the case of linear elliptic problems. The results also can be viewed in another aspect that since the obstacle problem with a general obstacle $\chi$ can be transformed into a problem with zero obstacle with nonhomogeneous Dirichlet boundary condition, for example see [21, the error estimator for general obstacle problem can be simplified to the error estimator for the zero obstacle. Generally, the error estimator for zero obstacle problem is simpler and is free of $\min / \max$ functions dealing with inconsistency of obstacle constraint.

The rest of the article is organized as follows. In the remaining part of this section, we introduce the model problem and some preliminaries. In section 2, we introduce some notation, define the discrete problem and derive some properties of the discrete solution. In section 3, we construct a post-processed discrete solution. We propose two methods for this purpose. One of them is by harmonic extension and the other by linear extension. Therein, we derive some error estimates for the discrete solution and its post-processed solution. In section 4, we present the a posteriori error analysis. In section 5, we derive error bounds that are simpler by rewriting general obstacle problem into a problem with zero obstacle. We present some numerical experiments in section 6 to illustrate the theoretical findings and finally conclude the article in section 7 .

Let $\Omega \in \mathbb{R}^{2}$ be a bounded polygonal domain with boundary denoted by $\partial \Omega$ (without slit). However the results on harmonic extension and the results in section 5 are applicable to three dimensional problems. We consider the obstacle $\chi \in C(\bar{\Omega}) \cap H^{1}(\Omega)$ satisfying $\left.\chi\right|_{\partial \Omega} \leq g$ a.e. on $\partial \Omega$, hereafter the function $g$ is assumed to be given and denotes the Dirichlet boundary data. Further, we assume that $g$ is the trace of a $H^{1}(\Omega)$ function. Define the closed and 
convex set by

$$
\mathcal{K}:=\left\{v \in H^{1}(\Omega): v \geq \chi \text { a.e. in } \Omega, \gamma_{0}(v)=g \text { on } \partial \Omega\right\},
$$

where $\gamma_{0}: H^{1}(\Omega) \rightarrow L^{2}(\partial \Omega)$ is the trace map, whose range is denoted by $\tilde{H}^{1 / 2}(\partial \Omega)$. Since $g \in \tilde{H}^{1 / 2}(\partial \Omega)$, there is some $\tilde{g} \in H^{1}(\Omega)$ with $\gamma_{0}(\tilde{g})=g$. Then it can be seen that the set $\mathcal{K}$ is nonempty as $\chi^{+}:=\max \{\chi, \tilde{g}\} \in \mathcal{K}$. The model problem for the discussion consists of finding $u \in \mathcal{K}$ such that

$$
a(u, v-u) \geq(f, v-u) \text { for all } v \in \mathcal{K},
$$

where $a(u, v):=(\nabla u, \nabla v)$ and $f \in L^{2}(\Omega)$ is a given function. Hereafter, $(\cdot, \cdot)$ denotes the $L^{2}(\Omega)$ inner-product while $\|\cdot\|$ denotes the $L^{2}(\Omega)$ norm. The existence of a unique solution to (1.1) follows from the result of Stampacchia [3, 22, 35].

For the rest of the discussions, we assume that the Dirichlet data $g \in \tilde{H}^{1 / 2}(\partial \Omega) \cap C(\partial \Omega)$. Define the Lagrange multiplier $\sigma \in H^{-1}(\Omega)$ by

$$
\langle\sigma, v\rangle=(f, v)-a(u, v) \text { for all } v \in H_{0}^{1}(\Omega),
$$

where $\langle\cdot, \cdot\rangle$ denotes the duality bracket of $H^{-1}(\Omega)$ and $H_{0}^{1}(\Omega)$. It follows from (1.2) and (1.1) that

$$
\langle\sigma, v-u\rangle \leq 0 \text { for all } v \in \mathcal{K} .
$$

Note that in the above equation (1.3), the test function $v-u \in H_{0}^{1}(\Omega)$ and hence the duality bracket $\langle\sigma, v-u\rangle$ is meaningful.

\section{Notation And Preliminaries}

Below, we list the notation that will be used throughout the article:

$$
\begin{aligned}
\mathcal{T}_{h} & :=\text { a regular simplicial triangulations of } \Omega \\
T & :=\text { a triangle of } \mathcal{T}_{h}, \quad|T|:=\text { area of } T \\
h_{T} & :=\text { diameter of } T, \quad h:=\max \left\{h_{T}: T \in \mathcal{T}_{h}\right\} \\
\mathcal{V}_{h}^{i} & :=\text { set of all vertices of } \mathcal{T}_{h} \text { that are in } \Omega \\
\mathcal{V}_{h}^{b} & :=\text { set of all vertices of } \mathcal{T}_{h} \text { that are on } \partial \Omega \\
\mathcal{V}_{T} & :=\text { set of three vertices of } T \\
\mathcal{E}_{h}^{i} & :=\text { set of all interior edges of } \mathcal{T}_{h} \\
\mathcal{E}_{h}^{b} & :=\text { set of all boundary edges of } \mathcal{T}_{h} \\
h_{e} & :=\text { length of an edge } e \in \mathcal{E}_{h} .
\end{aligned}
$$

We mean by regular triangulation that the triangles in $\mathcal{T}_{h}$ are shape-regular and there are no hanging nodes in $\mathcal{T}_{h}$. Each triangle $T$ in $\mathcal{T}_{h}$ is assumed to be closed. Define $\mathcal{T}_{h}^{i}$ to be the set of all triangles which do not share an edge with boundary $\partial \Omega$. Let $\mathcal{T}_{h}^{b}$ denote the remaining set of triangles, i.e., the set of all triangles $T \in \mathcal{T}_{h}$ which have an edge $e \in \mathcal{E}_{h}^{b}$ 
on its boundary $\partial T$. For simplicity assume that any $T \in \mathcal{T}_{h}^{b}$ shares at most one edge with boundary $\partial \Omega$, otherwise the triangle $T$ has no interior node.

In order to define the jump of discontinuous functions conveniently, define a broken Sobolev space

$$
H^{1}\left(\Omega, \mathcal{T}_{h}\right):=\left\{v \in L^{2}(\Omega): v_{T}=\left.v\right|_{T} \in H^{1}(T) \text { for all } T \in \mathcal{T}_{h}\right\} .
$$

For any $e \in \mathcal{E}_{h}^{i}$, there are two triangles $T_{+}$and $T_{-}$such that $e=\partial T_{+} \cap \partial T_{-}$. Let $n_{+}$be the unit normal of $e$ pointing from $T_{+}$to $T_{-}$, and $n_{-}=-n_{+}$, see Fig 5.1. For any $v \in H^{1}\left(\Omega, \mathcal{T}_{h}\right)$, we define the jump of $v$ on $e$ by

$$
\llbracket[v]:=v_{+}+v_{-},
$$

where $v_{ \pm}=\left.v\right|_{T_{ \pm}}$. Similarly define the jump of $w \in H^{1}\left(\Omega, \mathcal{T}_{h}\right)^{2}$ on $e \in \mathcal{E}_{h}^{i}$ by

$$
\llbracket w \rrbracket:=w_{-} \cdot n_{-}+w_{+} \cdot n_{+},
$$

where $w_{ \pm}=\left.w\right|_{T_{ \pm}}$. For any edge $e \in \mathcal{E}_{h}^{b}$, there is a triangle $T \in \mathcal{T}_{h}$ such that $e=\partial T \cap \partial \Omega$. For any $v \in H^{1}(T)$, we set on $e \in \mathcal{E}_{h}^{b}$

$$
\llbracket v \rrbracket:=v .
$$

2.1. Discrete Problem. We use the following linear finite element spaces $V_{h}$ and $V_{h}^{0}$ defined on $\mathcal{T}_{h}$ by

$$
V_{h}:=\left\{v_{h} \in H^{1}(\Omega):\left.v_{h}\right|_{T} \in \mathbb{P}_{1}(T) \text { for all } T \in \mathcal{T}_{h}\right\}
$$

and $V_{h}^{0}=V_{h} \cap H_{0}^{1}(\Omega)$, respectively. Define the discrete trace space $W_{h}$ by

$$
W_{h}:=\left\{w_{h} \in C(\partial \Omega):\left.v_{h}\right|_{e} \in \mathbb{P}_{1}(e) \text { for all } e \in \mathcal{E}_{h}^{b}\right\}
$$

Let $g_{h} \in W_{h}$ be the nodal interpolation of $g$, i.e. $g_{h}(z)=g(z)$ for all $z \in \mathcal{V}_{h}^{b}$. Define the discrete set

$$
\mathcal{K}_{h}:=\left\{v_{h} \in V_{h}: v_{h}(z) \geq \chi(z) \text { for all } z \in \mathcal{V}_{h}^{i}, \quad v_{h}=g_{h} \text { on } \partial \Omega\right\} .
$$

The discrete problem consists of finding $u_{h} \in \mathcal{K}_{h}$ such that

$$
a\left(u_{h}, v_{h}-u_{h}\right) \geq\left(f, v_{h}-u_{h}\right) \text { for all } v_{h} \in \mathcal{K}_{h} .
$$

Similar to the case of the continuous problem (1.1), it can easily be shown that the discrete problem (2.2) has a unique solution. Below, we derive some properties of the solution $u_{h}$.

Note that for any $z \in \mathcal{V}_{h}^{i}$ and the corresponding Lagrange basis function (hat function) $\psi_{z}$ defined by

$$
q \in \mathcal{V}_{h}^{i}, \quad \psi_{z}(q):= \begin{cases}1 & \text { if } z=q, \\ 0 & \text { otherwise }\end{cases}
$$

we have $v_{h}=u_{h}+\psi_{z} \in \mathcal{K}_{h}$. Then (2.2) implies that

$$
a\left(u_{h}, \psi_{z}\right) \geq\left(f, \psi_{z}\right) \text { for all } z \in \mathcal{V}_{h}^{i} .
$$


Furthermore if $u_{h}(z)>\chi(z)$ for any $z \in \mathcal{V}_{h}^{i}$, it is easy to prove by considering $v_{h}^{ \pm}=u_{h} \pm \epsilon \psi_{z} \in$ $\mathcal{K}_{h}$ (for sufficiently small $\epsilon>0$ ) in (2.2) that

$$
a\left(u_{h}, \psi_{z}\right)=\left(f, \psi_{z}\right) \text { for all } z \in\left\{z \in \mathcal{V}_{h}^{i}: u_{h}(z)>\chi(z)\right\} .
$$

As in the case of continuous problem, we define the discrete Lagrange multiplier $\sigma_{h} \in V_{h}^{0}$ by

$$
\left\langle\sigma_{h}, v\right\rangle_{h}=(f, v)-a\left(u_{h}, v\right) \quad \forall v \in V_{h}^{0},
$$

where

$$
\langle w, v\rangle_{h}=\sum_{T \in \mathcal{T}_{h}} \frac{|T|}{3} \sum_{z \in \mathcal{V}_{T}} w(z) v(z)
$$

which is the well-known mass lumping formula.

Using (2.3)-(2.4), we deduce that

$$
\begin{aligned}
& \sigma_{h}(z) \geq 0 \quad \forall z \in \mathcal{V}_{h}, \\
& \sigma_{h}(z)=0 \quad \text { if } u_{h}(z)>\chi(z), \quad z \in \mathcal{V}_{h}^{i} .
\end{aligned}
$$

The following error estimate for the lumped-mass numerical integration is well-known, 44, 24]: For any $T \in \mathcal{T}_{h}$ and $v_{h}, w_{h} \in V_{h}$, there holds

$$
\left|\frac{|T|}{3} \sum_{z \in \mathcal{V}_{T}} v_{h}(z) w_{h}(z)-\int_{T} v_{h} w_{h} d x\right| \leq C h_{T}\left\|\nabla v_{h}\right\|_{L_{2}(T)}\left\|\nabla w_{h}\right\|_{L_{2}(T)} .
$$

\section{Post-Processed Solution}

Since the discrete problem (2.2) uses the approximate Dirichlet data $g_{h}$ which is not equal to the given data $g$, the numerical scheme can be treated as a nonconforming scheme with respect to the Dirichlet data. In this section, we propose two post-processing methods to construct an intermediate solution $\tilde{u}_{h}$ (which can be treated as a post-processing of $u_{h}$ ) constructed with the help of $u_{h}$ and $g$ as follows. For $T \in \mathcal{T}_{h}^{i}$, define $\tilde{u}_{h}$ to be the same as $u_{h}$ on $T$. For $T \in \mathcal{T}_{h}^{b}$, first define $u_{h}^{*}$ on $\partial T$ ( the boundary of $T$ ) by

$$
u_{h}^{*}(x, y):= \begin{cases}g(x, y) & \text { if }(x, y) \in \partial \Omega \\ u_{h}(x, y) & \text { if }(x, y) \in \partial T \backslash \partial \Omega .\end{cases}
$$

Then define $\tilde{u}_{h}$ to be some $H^{1}(T)$ extension of $u_{h}^{*} \in C(\partial T)$ to $T$ such that $\left.\tilde{u}_{h}\right|_{\partial T}=\left.u_{h}^{*}\right|_{\partial T}$ and $\left.\tilde{u}_{h}\right|_{T}=\left.u_{h}\right|_{T}$ for all $T \in \mathcal{T}_{h}^{i}$. By the construction $\tilde{u}_{h} \in H^{1}(\Omega)$ and $u_{h}-\tilde{u}_{h} \equiv 0$ on any triangle $T \in \mathcal{T}_{h}^{i}$.

In general, there will be many choices of $\tilde{u}_{h}$. In the following we will present two methods for constructing it. One approach is by using the Harmonic extension and the other is by linear interpolation. We discuss this in two dimensions context and note that the similar extension in three dimension is quite similar. 
Method 1. (Harmonic Extension): Let $T \in \mathcal{T}_{h}^{b}$ and $u_{h}^{*} \in C(\partial T)$ be defined by (3.1). Then define $\tilde{u}_{h} \in H^{1}(T)$ to be the weak solution of

$$
\begin{aligned}
\tilde{u}_{h} & =u_{h}^{*} \text { on } \partial T, \\
\Delta \tilde{u}_{h} & =0 \text { in } T .
\end{aligned}
$$

By the stability of elliptic problems and scaling, we find that

$$
\left\|\tilde{u}_{h}\right\|_{H^{1}(T)} \leq C\left\|u_{h}^{*}\right\|_{H^{1 / 2}(\partial T)}
$$

where the constant $C$ is independent of $T$. Note that $\tilde{u}_{h}-u_{h}$ satisfies

$$
\begin{aligned}
\tilde{u}_{h}-u_{h} & =u_{h}^{*}-u_{h} \text { on } \partial T, \\
\Delta\left(\tilde{u}_{h}-u_{h}\right) & =0 \text { in } T .
\end{aligned}
$$

We can show by the stability of elliptic problems and scaling that

$$
\left\|\tilde{u}_{h}-u_{h}\right\|_{H^{1}(T)} \leq C\left\|u_{h}^{*}-u_{h}\right\|_{H^{1 / 2}(\partial T)} .
$$

In addition if $g \in H^{1}(e)$ where $e=\partial \Omega \cap \partial T$, then by the approximation property of Lagrange interpolation, see [15], we find

$$
\left\|u_{h}^{*}-u_{h}\right\|_{H^{1 / 2}(\partial T)} \leq C h_{T}^{1 / 2}\left|g-g_{h}\right|_{H^{1}(e)},
$$

and hence

$$
\left\|\tilde{u}_{h}-u_{h}\right\|_{H^{1}(T)} \leq C h_{T}^{1 / 2}\left|g-g_{h}\right|_{H^{1}(e)} .
$$

Further since the triangle $T$ is convex and $g \in H^{1}(e)$, by the elliptic regularity theory $\tilde{u}_{h} \in H^{3 / 2}(T) \subset C(\bar{T})$. Then the weak maximum principle for harmonic functions implies that

$$
\min _{z \in T} \tilde{u}_{h}(z)=\min _{z \in \partial T} u_{h}^{*}(z) .
$$

We deduce the following proposition on the harmonic extension:

Proposition 3.1. Let $T \in \mathcal{T}_{h}^{b}$ and $e$ be an edge of $T$ with $e \in \mathcal{E}_{h}^{b}$. Let $\tilde{u}_{h}$ be the harmonic extension of $u_{h}^{*}$ defined by (3.2) -(3.3). Then, if $g \in H^{1}(e)$, there holds

$$
\left\|\tilde{u}_{h}-u_{h}\right\|_{H^{1}(T)} \leq C h_{T}^{1 / 2}\left|g-g_{h}\right|_{H^{1}(e)},
$$

and furthermore

$$
\min _{z \in T} \tilde{u}_{h}(z)=\min _{z \in \partial T} u_{h}^{*}(z) .
$$

Next we proceed to present another approach by linear extension (linear interpolation). Method 2. (Linear Extension): Let $T \in \mathcal{T}_{h}^{b}$ and $u_{h}^{*} \in C(\partial T)$ be defined by (3.1). Let $e \in \mathcal{E}_{h}^{b}$ be such that $e \subset \partial T$. We will construct $\tilde{u}_{h}$ by connecting through line segments using the values of $u_{h}^{*}$ along the lines perpendicular to $e$. Let $z=(x, y)$ be an interior point of $T$ and $\ell$ be the line that is orthogonal to $e$ passing through $z$, as it shown in the Figure 3.1 . Let $z_{1}=\left(x_{0}, y_{0}\right)$ and $z_{2}=\left(x_{1}, y_{1}\right)$ be the points of intersection of $\ell$ with $\partial T$. Then $\tilde{u}_{h}$ is defined 
on $\ell$ as a linear polynomial assuming the values $u_{h}^{*}\left(x_{0}, y_{0}\right)$ and $u_{h}^{*}\left(x_{1}, y_{1}\right)$ at $z_{1}=\left(x_{0}, y_{0}\right)$ and $z_{2}=\left(x_{1}, y_{1}\right)$, respectively.

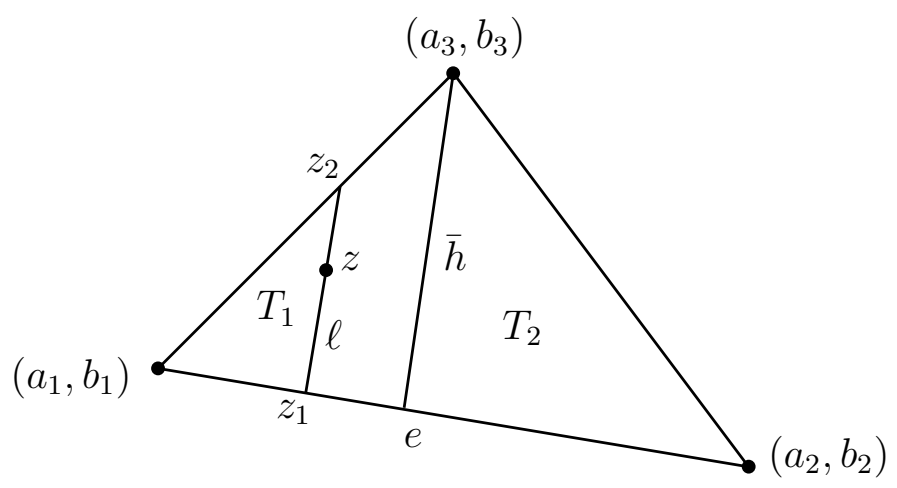

Figure 3.1. Subdivision of $T=T_{1} \cup T_{2}$

We divide the triangle $T$ as $T=T_{1} \cup T_{2}$, where $T_{1}$ and $T_{2}$ are sub triangles of $T$ separated by the height $\bar{h}$ as shown in the Figure 3.1. Then define $\tilde{u}_{h}$ on $T$ by

$$
\tilde{u}_{h}(x, y):=\frac{h_{2} u_{h}^{*}\left(x_{0}, y_{0}\right)+h_{1} u_{h}^{*}\left(x_{1}, y_{1}\right)}{h_{1}+h_{2}} \quad \text { for }(x, y) \in T^{\circ},
$$

where $T^{\circ}$ is the interior of $T$,

$$
\begin{aligned}
& x_{0}=\frac{\left(a_{1}-a_{2}\right)\left(\left(a_{1}-a_{2}\right) x+\left(b_{1}-b_{2}\right) y\right)+\left(b_{1}-b_{2}\right)\left(b_{1} a_{2}-b_{2} a_{1}\right)}{\left(a_{1}-a_{2}\right)^{2}+\left(b_{1}-b_{2}\right)^{2}}, \\
& y_{0}=\frac{\left(b_{2}-b_{1}\right)\left(\left(a_{2}-a_{1}\right) x+\left(b_{2}-b_{1}\right) y\right)+\left(a_{2}-a_{1}\right)\left(b_{1} a_{2}-b_{2} a_{1}\right)}{\left(a_{1}-a_{2}\right)^{2}+\left(b_{1}-b_{2}\right)^{2}}, \\
& x_{1}=\frac{\left(a_{i}-a_{3}\right)\left(\left(a_{1}-a_{2}\right) x+\left(b_{1}-b_{2}\right) y\right)+\left(b_{1}-b_{2}\right)\left(b_{i} a_{3}-b_{3} a_{i}\right)}{\left(a_{1}-a_{2}\right)\left(a_{i}-a_{3}\right)+\left(b_{1}-b_{2}\right)\left(b_{i}-b_{3}\right)}, \\
& y_{1}=\frac{\left(b_{i}-b_{3}\right)\left(\left(a_{1}-a_{2}\right) x+\left(b_{1}-b_{2}\right) y\right)+\left(a_{1}-a_{2}\right)\left(b_{3} a_{i}-b_{i} a_{3}\right)}{\left(a_{1}-a_{2}\right)\left(a_{i}-a_{3}\right)+\left(b_{1}-b_{2}\right)\left(b_{i}-b_{3}\right)} \\
& h_{1}=\frac{\left|\left(b_{2}-b_{1}\right) x+\left(a_{1}-a_{2}\right) y+\left(b_{1} a_{2}-b_{2} a_{1}\right)\right|}{\sqrt{\left(b_{2}-b_{1}\right)^{2}+\left(a_{2}-a_{1}\right)^{2}}}
\end{aligned}
$$




$$
h_{2}=\sqrt{\left(b_{1}-b_{2}\right)^{2}+\left(a_{1}-a_{2}\right)^{2}} \frac{\left|\left(a_{i}-a_{3}\right) y-\left(b_{i}-b_{3}\right) x+\left(b_{i} a_{3}-b_{3} a_{i}\right)\right|}{\left|\left(a_{1}-a_{2}\right)\left(a_{i}-a_{3}\right)+\left(b_{1}-b_{2}\right)\left(b_{i}-b_{3}\right)\right|},
$$

where $|\cdot|$ denotes the absolute value and

$i= \begin{cases}1 & \text { if }(x, y) \in T_{1}, \\ 2 & \text { if }(x, y) \in T_{2} .\end{cases}$

Note that $h_{1}$ is the distance between $z=(x, y)$ and $z_{1}=\left(x_{0}, y_{0}\right)$; and $h_{2}$ is the distance between $z=(x, y)$ and $z_{2}\left(x_{1}, y_{1}\right)$.

Again by the construction, it is immediate to see that

$$
\min _{z \in T} \tilde{u}_{h}(z)=\min _{z \in \partial T} u_{h}^{*}(z) .
$$

In the rest of this section, we assume that $\left.g\right|_{e} \in H^{1}(e)$ and we establish the following estimate:

Theorem 3.2. Let $T \in \mathcal{T}_{h}^{b}$ and define $\tilde{u}_{h}$ by (3.6), a linear extension of $u_{h}^{*}$ given by (3.1). Let $e \in \mathcal{E}_{h}^{b}$ be an edge of $T$ and assume that $\left.g\right|_{e} \in H^{1}(e)$. Then there holds

$$
\left\|\nabla\left(\tilde{u}_{h}-u_{h}\right)\right\|_{L^{2}(T)} \leq C h_{e}^{\frac{1}{2}}\left\|\left(g^{\prime}-g_{h}^{\prime}\right)\right\|_{L^{2}(e)},
$$

where $C$ is a positive constant, $e \in \mathcal{E}_{h}^{b}$ an edge of $T$ and $g^{\prime}$ (resp. $g_{h}^{\prime}$ ) denotes the tangential derivative of $g$ (resp. $g_{h}$ ) along $e$.

In subsequent discussion, we prove two lemmas before proving the theorem. Note that $\tilde{u}_{h} \in L^{2}(T)$ for all $T \in \mathcal{T}_{h}^{b}$. Therefore it defines a distribution via

$$
\tilde{u}_{h}(\phi)=\int_{T} \tilde{u}_{h} \phi d x \quad \forall \phi \in \mathcal{D}(T),
$$

where $\mathcal{D}(T)$ is the space of all $C^{\infty}$ functions having compact support in $T$. The distribution derivative $\partial \tilde{u}_{h} / \partial x_{i}$ for $(i=1,2)$ is given by [34,

$$
\frac{\partial \tilde{u}_{h}}{\partial x_{i}}(\phi)=-\int_{T} \tilde{u}_{h} \frac{\partial \phi}{\partial x_{i}} d x \quad \forall \phi \in \mathcal{D}(T) .
$$

Further, we have

$$
\frac{\partial\left(\tilde{u}_{h}-u_{h}\right)}{\partial x_{i}}(\phi)=-\int_{T}\left(\tilde{u}_{h}-u_{h}\right) \frac{\partial \phi}{\partial x_{i}} d x \quad \forall \phi \in \mathcal{D}(T) .
$$

Since $\left.u_{h}\right|_{T} \in P_{1}(T)$, we note that

$$
u_{h}(x, y)=\frac{h_{2} u_{h}\left(x_{0}, y_{0}\right)+h_{1} u_{h}\left(x_{1}, y_{1}\right)}{h_{1}+h_{2}} \quad \text { for }(x, y) \in T^{\circ},
$$

and

$$
\left(\tilde{u}_{h}-u_{h}\right)(x, y)=\left(\frac{h_{2}}{h_{1}+h_{2}}\right)\left(g-g_{h}\right)\left(x_{0}, y_{0}\right) \text {. }
$$


From (3.10), we can treat the differentiation on $\tilde{u}_{h}-u_{h}$ under the integral (in the sense of a.e.) and write

$$
\begin{aligned}
\nabla\left(\tilde{u}_{h}-u_{h}\right)(x, y)=( & \left.\frac{h_{1} \nabla h_{2}-h_{2} \nabla h_{1}}{\left(h_{1}+h_{2}\right)^{2}}\right)\left(g-g_{h}\right)\left(x_{0}, y_{0}\right) \\
& +\left(\frac{h_{2}}{h_{1}+h_{2}}\right) \nabla\left(\left(g-g_{h}\right)\left(x_{0}, y_{0}\right)\right) .
\end{aligned}
$$

Using the formula for $h_{1}$ and $h_{2}$, we find

$$
\begin{aligned}
& \nabla h_{1}=\left(\frac{\left(b_{2}-b_{1}\right)}{\sqrt{\left(b_{1}-b_{2}\right)^{2}+\left(a_{1}-a_{2}\right)^{2}}}, \frac{\left(a_{1}-a_{2}\right)}{\sqrt{\left(b_{1}-b_{2}\right)^{2}+\left(a_{1}-a_{2}\right)^{2}}}\right), \\
& \nabla h_{2}=\frac{\sqrt{\left(b_{1}-b_{2}\right)^{2}+\left(a_{1}-a_{2}\right)^{2}}}{\left|\left(a_{1}-a_{2}\right)\left(a_{i}-a_{3}\right)+\left(b_{1}-b_{2}\right)\left(b_{i}-b_{3}\right)\right|}\left(\left(b_{i}-b_{3}\right),\left(a_{i}-a_{3}\right)\right),
\end{aligned}
$$

and then

$$
\begin{aligned}
\left|\nabla h_{1} \cdot \nabla h_{2}\right| & =1 \\
\left|\nabla h_{1} \cdot \nabla h_{1}\right| & =1 \\
\nabla h_{2} \cdot \nabla h_{2} & =\frac{\left(\left(b_{1}-b_{2}\right)^{2}+\left(a_{1}-a_{2}\right)^{2}\right)\left(\left(b_{i}-b_{3}\right)^{2}+\left(a_{i}-a_{3}\right)^{2}\right)}{\left|\left(a_{1}-a_{2}\right)\left(a_{i}-a_{3}\right)+\left(b_{1}-b_{2}\right)\left(b_{i}-b_{3}\right)\right|^{2}}=\frac{1}{\cos ^{2}\left(\theta_{i}\right)},
\end{aligned}
$$

where, $\theta_{i}$ is the interior angle of $T$ at $\left(a_{i}, b_{i}\right)$ for $i=1,2$. Since the triangulation is regular, the minimal angle in the triangulation $\mathcal{T}_{h}$ is bounded below by some angle $\theta_{0}$. Therefore $\theta_{i} \leq \pi / 2-\theta_{0}$ and $\cos \left(\theta_{i}\right) \geq \cos \left(\pi / 2-\theta_{0}\right) \geq C$ for some positive constant $C$ uniformly. Further, we find by the chain rule

$$
\begin{aligned}
& \frac{\partial\left(\left(g-g_{h}\right)\left(x_{0}, y_{0}\right)\right)}{\partial x}=\frac{t_{1}}{h_{e}^{2}}\left(t_{1} \frac{\partial\left(g-g_{h}\right)}{\partial x_{0}}\left(x_{0}, y_{0}\right)+t_{2} \frac{\partial\left(g-g_{h}\right)}{\partial y_{0}}\left(x_{0}, y_{0}\right)\right) \\
& \frac{\partial\left(\left(g-g_{h}\right)\left(x_{0}, y_{0}\right)\right)}{\partial y}=\frac{t_{2}}{h_{e}^{2}}\left(t_{1} \frac{\partial\left(g-g_{h}\right)}{\partial x_{0}}\left(x_{0}, y_{0}\right)+t_{2} \frac{\partial\left(g-g_{h}\right)}{\partial y_{0}}\left(x_{0}, y_{0}\right)\right)
\end{aligned}
$$

where $h_{e}=\sqrt{\left(a_{1}-a_{2}\right)^{2}+\left(b_{1}-b_{2}\right)^{2}}, t_{1}=a_{1}-a_{2}$ and $t_{2}=b_{1}-b_{2}$. Let $\mathbf{t}=\left(\hat{t}_{1}, \hat{t}_{2}\right)=$ $\left(t_{1}, t_{2}\right) / h_{e}$. Then $\mathbf{t}$ is the unit tangent vector to the edge $e$ in the direction of $\left(t_{1}, t_{2}\right)$. It is now easy to see that

$$
\left|\nabla\left(g-g_{h}\right)\left(x_{0}, y_{0}\right)\right|=\left|\hat{t}_{1} \frac{\partial\left(g-g_{h}\right)}{\partial x_{0}}\left(x_{0}, y_{0}\right)+\hat{t}_{2} \frac{\partial\left(g-g_{h}\right)}{\partial y_{0}}\left(x_{0}, y_{0}\right)\right| .
$$

The term on the right hand side of the above inequality is nothing but the tangential derivative of $\left(g-g_{h}\right)$ in the direction of $\left(t_{1}, t_{2}\right)$ on the edge $e$. We now prove the following lemma: 
Lemma 3.3. Let $x_{0}(x, y)=x_{0}$ and $y_{0}(x, y)=y_{0}$ be defined as in (3.7) and (3.8), respectively. Then for $T \in \mathcal{T}_{h}^{b}$ and $e=\partial T \cap \partial \Omega$, there holds

$$
\int_{T} \mid \nabla\left(g-g_{h}\right)\left(x_{0}(x, y),\left.y_{0}(x, y)\right|^{2} d x d y \leq C h_{e}\left\|\left(g^{\prime}-g_{h}^{\prime}\right)\right\|_{L^{2}(e)}^{2},\right.
$$

where $g^{\prime}$ (resp. $g_{h}^{\prime}$ ) denotes the tangential derivative of $g$ (resp. $g_{h}$ ) along e.

Proof. From the identity (3.12), we find

$$
\begin{aligned}
\int_{T} \mid & \nabla\left(g-g_{h}\right)\left(x_{0}(x, y),\left.y_{0}(x, y)\right|^{2} d x d y\right. \\
& =\int_{T}\left|\hat{t}_{1} \frac{\partial\left(g-g_{h}\right)}{\partial x_{0}}\left(x_{0}(x, y), y_{0}(x, y)\right)+\hat{t}_{2} \frac{\partial\left(g-g_{h}\right)}{\partial y_{0}}\left(x_{0}(x, y), y_{0}(x, y)\right)\right|^{2} d x d y \\
& =\int_{T}\left|\left(g-g_{h}\right)^{\prime}\left(x_{0}(x, y), y_{0}(x, y)\right)\right|^{2} d x d y .
\end{aligned}
$$

Now the proof follows from the observation that the integrand is constant along the lines that are orthogonal to $e$.

We further prove the following lemma:

Lemma 3.4. Assume the hypothesis of Lemma 3.3, then there holds

$$
\int_{T}\left|\left(\frac{h_{1} \nabla h_{2}-h_{2} \nabla h_{1}}{\left(h_{1}+h_{2}\right)^{2}}\right)\left(g-g_{h}\right)\left(x_{0}(x, y), y_{0}(x, y)\right)\right|^{2} d x d y \leq C h_{e}\left\|\left(g^{\prime}-g_{h}^{\prime}\right)\right\|_{L^{2}(e)}^{2} .
$$

Proof. For simplicity denote $x_{0}(x, y)$ by $x_{0}$ and $y_{0}(x, y)=y_{0}$. Then

$$
\begin{aligned}
\int_{T} \mid & \left.\left(\frac{h_{1} \nabla h_{2}-h_{2} \nabla h_{1}}{\left(h_{1}+h_{2}\right)^{2}}\right)\left(g-g_{h}\right)\left(x_{0}, y_{0}\right)\right|^{2} d x d y \\
& =\int_{T}\left(\frac{\left(\nabla h_{2} \cdot \nabla h_{2}\right) h_{1}^{2}+\left(\nabla h_{1} \cdot \nabla h_{1}\right) h_{2}^{2}-2 h_{2} h_{1} \nabla h_{2} \cdot \nabla h_{1}}{\left(h_{1}+h_{2}\right)^{4}}\right)\left(g-g_{h}\right)^{2}\left(x_{0}, y_{0}\right) d x d y \\
& \leq \int_{T}\left(\frac{\left(\cos \theta_{i}\right)^{-2} h_{1}^{2}+h_{2}^{2}+2 h_{2} h_{1}}{\left(h_{1}+h_{2}\right)^{4}}\right)\left(g-g_{h}\right)^{2}\left(x_{0}, y_{0}\right) d x d y \\
& \leq \frac{1}{\left(\cos \theta_{i}\right)^{2}} \int_{T}\left(\frac{h_{1}^{2}+h_{2}^{2}+2 h_{2} h_{1}}{\left(h_{1}+h_{2}\right)^{4}}\right)\left(g-g_{h}\right)^{2}\left(x_{0}, y_{0}\right) d x d y \\
& \leq \frac{1}{\left(\cos \theta_{i}\right)^{2}} \int_{T} \frac{\left(g-g_{h}\right)^{2}\left(x_{0}, y_{0}\right)}{\left(h_{1}+h_{2}\right)^{2}} d x d y \\
& =\frac{1}{\left(\cos \theta_{i}\right)^{2}}\left(\int_{T_{1}} \frac{\left(g-g_{h}\right)^{2}\left(x_{0}, y_{0}\right)}{\left(h_{1}+h_{2}\right)^{2}} d x d y+\int_{T_{2}} \frac{\left(g-g_{h}\right)^{2}\left(x_{0}, y_{0}\right)}{\left(h_{1}+h_{2}\right)^{2}} d x d y\right)
\end{aligned}
$$


Without loss of generality, assume that the boundary edge is parallel to the $x$-axis and consider the integral on $T_{1}$.

$$
\begin{aligned}
\int_{T_{1}} \frac{\left(g-g_{h}\right)^{2}\left(x_{0}, y_{0}\right)}{\left(h_{1}+h_{2}\right)^{2}} d x d y & =\int_{a_{1}}^{a_{3}} \int_{b_{1}}^{b_{1}+\left(\frac{b_{3}-b_{1}}{a_{3}-a_{1}}\right)} \frac{\left(x-a_{1}\right)}{\left(\left(\frac{b_{3}-b_{1}}{a_{3}-a_{1}}\right)\left(x-a_{1}\right)\right)^{2}} d x d y \\
& =\left(\frac{a_{3}-a_{1}}{b_{3}-b_{1}}\right) \int_{a_{1}}^{a_{3}} \frac{\left(g-g_{h}\right)^{2}\left(x, b_{1}\right)}{\left(x-a_{1}\right)} d x .
\end{aligned}
$$

Since $g \in H^{1}(\bar{e}), g$ is absolutely continuous on $e$ [34, Theorem 2.1.2]. Then as $(g-$ $\left.g_{h}\right)\left(a_{1}, b_{1}\right)=0$, we find using a property of absolutely continuous functions [36, Theorem 14 on page 110] that for any $x \in\left(a_{1}, a_{3}\right)$,

$$
\left(g-g_{h}\right)\left(x, b_{1}\right)=\int_{a_{1}}^{x}\left(g-g_{h}\right)^{\prime}\left(t, b_{1}\right) d t .
$$

Subsequently, there holds

$$
\left|\left(g-g_{h}\right)\left(x, b_{1}\right)\right|^{2} \leq\left|x-a_{1}\right|\left\|\left(g-g_{h}\right)^{\prime}\right\|_{L_{2}(e)}^{2} .
$$

Also since $\left(a_{3}-a_{1}\right) /\left(b_{3}-b_{1}\right) \leq C$ for some constant $C$ which is independent of $T$, we obtain

$$
\int_{T_{1}} \frac{\left(g-g_{h}\right)^{2}\left(x_{0}, y_{0}\right)}{\left(h_{1}+h_{2}\right)^{2}} d x d y \leq\left(\frac{a_{3}-a_{1}}{b_{3}-b_{1}}\right)\left(a_{3}-a_{1}\right)\left\|\left(g-g_{h}\right)^{\prime}\right\|_{L_{2}(e)}^{2} \leq C h_{e}\left\|\left(g-g_{h}\right)^{\prime}\right\|_{L_{2}(e)}^{2} .
$$

Using the same arguments, we obtain the similar estimate for $T_{2}$. Finally using the fact that $\cos \left(\theta_{i}\right) \geq C$ for some positive constant, we conclude the proof.

Proof of Theorem 3.2, Using the equation (3.11) and since

$$
\left|\frac{h_{2}}{h_{1}+h_{2}}\right| \leq 1
$$

we complete the proof by using the triangle inequality, Lemma 3.3 and Lemma 3.4 .

\section{A posteriori Error Analysis}

In this section, we derive a reliable and efficient a posteriori error estimator. The error analysis can conveniently be derived by using an appropriate residual. To this end, define the residual $\mathcal{R}_{h}: H_{0}^{1}(\Omega) \rightarrow \mathbb{R}$ by

$$
\mathcal{R}_{h}(v):=(f, v)-a\left(\tilde{u}_{h}, v\right)-\left(\sigma_{h}, v\right)
$$

or equivalently, define

$$
\mathcal{R}_{h}(v):=a\left(u-\tilde{u}_{h}, v\right)+\left\langle\sigma-\sigma_{h}, v\right\rangle,
$$

where $\tilde{u}_{h}$ is either the harmonic extension or the linear extension as in Section 3 . The following lemma establishes a relation between the residual and the errors. 
Lemma 4.1. There holds

$$
\left\|\nabla\left(u-u_{h}\right)\right\|^{2}+\left\|\sigma-\sigma_{h}\right\|_{-1}^{2} \leq 6\left\|\mathcal{R}_{h}\right\|_{-1}^{2}-8\left\langle\sigma-\sigma_{h}, u-\tilde{u}_{h}\right\rangle+2\left\|\nabla\left(u_{h}-\tilde{u}_{h}\right)\right\|^{2} .
$$

Proof. From (4.2) and Young's inequality, we find

$$
\begin{aligned}
\left\|\nabla\left(u-\tilde{u}_{h}\right)\right\|^{2} & =a\left(u-\tilde{u}_{h}, u-\tilde{u}_{h}\right)=\mathcal{R}_{h}\left(u-\tilde{u}_{h}\right)-\left\langle\sigma-\sigma_{h}, u-\tilde{u}_{h}\right\rangle \\
& \leq\left\|\mathcal{R}_{h}\right\|_{-1}\left\|\nabla\left(u-\tilde{u}_{h}\right)\right\|-\left\langle\sigma-\sigma_{h}, u-\tilde{u}_{h}\right\rangle \\
& \leq \frac{1}{2}\left\|\mathcal{R}_{h}\right\|_{-1}^{2}+\frac{1}{2}\left\|\nabla\left(u-\tilde{u}_{h}\right)\right\|^{2}-\left\langle\sigma-\sigma_{h}, u-\tilde{u}_{h}\right\rangle,
\end{aligned}
$$

which proves

$$
\left\|\nabla\left(u-\tilde{u}_{h}\right)\right\|^{2} \leq\left\|\mathcal{R}_{h}\right\|_{-1}^{2}-2\left\langle\sigma-\sigma_{h}, u-\tilde{u}_{h}\right\rangle .
$$

Then the triangle inequality and Young's inequality imply

$$
\begin{aligned}
\left\|\nabla\left(u-u_{h}\right)\right\|^{2} & \leq 2\left\|\nabla\left(u-\tilde{u}_{h}\right)\right\|^{2}+2\left\|\nabla\left(u_{h}-\tilde{u}_{h}\right)\right\|^{2} \\
& \leq 2\left\|\mathcal{R}_{h}\right\|_{-1}^{2}-4\left\langle\sigma-\sigma_{h}, u-\tilde{u}_{h}\right\rangle+2\left\|\nabla\left(u_{h}-\tilde{u}_{h}\right)\right\|^{2} .
\end{aligned}
$$

Again from (4.2), we find

$$
\left\|\sigma-\sigma_{h}\right\|_{-1} \leq\left\|\mathcal{R}_{h}\right\|_{-1}+\left\|\nabla\left(u-\tilde{u}_{h}\right)\right\|,
$$

then by Young's inequality and (4.3),

$$
\begin{aligned}
\left\|\sigma-\sigma_{h}\right\|_{-1}^{2} & \leq 2\left\|\mathcal{R}_{h}\right\|_{-1}^{2}+2\left\|\nabla\left(u-\tilde{u}_{h}\right)\right\|^{2} \\
& \leq 4\left\|\mathcal{R}_{h}\right\|_{-1}^{2}-4\left\langle\sigma-\sigma_{h}, u-\tilde{u}_{h}\right\rangle .
\end{aligned}
$$

The proof then follows by combining the estimates in (4.4)-(4.5).

In order to derive the error analysis, we define the following error estimators :

$$
\begin{aligned}
\eta_{f, T} & :=h_{T}\left\|f-\sigma_{h}\right\|_{L_{2}(T)}, \\
\eta_{u_{h}, e} & :=h_{e}^{1 / 2}\left\|\left[\nabla \nabla u_{h}\right]\right\|_{L_{2}(e)}, \\
\eta_{\sigma, T} & :=h_{T}^{2}\left\|\nabla \sigma_{h}\right\|_{L_{2}(T)},
\end{aligned}
$$

and

$$
\eta_{f}^{2}:=\sum_{T \in \mathcal{T}_{h}} \eta_{f, T}^{2}, \quad \eta_{J}^{2}:=\sum_{e \in \mathcal{E}_{h}^{i}} \eta_{u_{h}, e}^{2}, \quad \eta_{\sigma}^{2}:=\sum_{T \in \mathcal{T}_{h}} \eta_{\sigma, T}^{2}, \quad \eta_{g}^{2}:=\left\|\nabla\left(u_{h}-\tilde{u}_{h}\right)\right\|^{2},
$$

and finally define

$$
\eta^{2}:=\eta_{f}^{2}+\eta_{J}^{2}+\eta_{\sigma}^{2}+\eta_{g}^{2}
$$

In the following lemma, we estimate the dual norm of the residual $\mathcal{R}_{h}$.

Lemma 4.2. There holds

$$
\left\|\mathcal{R}_{h}\right\|_{-1} \leq C \eta
$$


Proof. From (4.1) and (2.5), we note that

$$
\mathcal{R}_{h}(v)=\left(f, v-v_{h}\right)-a\left(u_{h}, v-v_{h}\right)-\left(\sigma_{h}, v-v_{h}\right)+\left\langle\sigma_{h}, v_{h}\right\rangle_{h}-\left(\sigma_{h}, v_{h}\right)+a\left(u_{h}-\tilde{u}_{h}, v\right),
$$

for any $v_{h} \in V_{h}$. Let $v_{h}$ be the Clement interpolation of $v$. Then the proof now follows by integration by parts, the approximation properties of $v_{h}$ and the estimate in (2.9).

It is now remaining to estimate $-\left\langle\sigma-\sigma_{h}, u-\tilde{u}_{h}\right\rangle$. The error estimator depends on the following sets defined by

$$
\begin{gathered}
\mathbb{F}_{h}:=\left\{T \in \mathcal{T}_{h}: \exists z_{1}, z_{2} \in \mathcal{V}_{T} \text { such that } u_{h}\left(z_{1}\right)=\chi\left(z_{1}\right) \text { and } u_{h}\left(z_{2}\right)>\chi\left(z_{2}\right)\right\} \\
\mathbb{C}_{h}:=\left\{T \in \mathcal{T}_{h}: \text { For all } z \in \mathcal{V}_{T}, u_{h}(z)=\chi(z)\right\}
\end{gathered}
$$

and

$$
\mathbb{N}_{h}:=\left\{T \in \mathcal{T}_{h}: \text { For all } z \in \mathcal{V}_{T}, u_{h}(z)>\chi(z)\right\}
$$

We call the sets $\mathbb{F}_{h}, \mathbb{C}_{h}$ and $\mathbb{N}_{h}$ as free boundary set, contact set and non-contact set, respectively. We also define

$$
\partial \mathbb{F}_{h}^{i}:=\left\{e \in \mathcal{E}_{h}^{i}: e \in \mathcal{E}_{z}, z \in \mathcal{V}_{T} \text { with }\left.u_{h}\right|_{T}(z)=\chi_{h}(z), T \in \mathbb{F}_{h}\right\},
$$

the set of all interior edges that share a vertex of a triangle in $\mathbb{F}_{h}$.

We prove the following lemma in the spirit of [44, 24]:

Lemma 4.3. Let $\chi_{h} \in V_{h}$ be the Lagrange interpolation of $\chi$. Then there holds

$$
\begin{aligned}
\left\langle\sigma-\sigma_{h}, u-\tilde{u}_{h}\right\rangle & \geq-\epsilon\left\|\sigma-\sigma_{h}\right\|_{-1}^{2}-\epsilon\left\|\nabla\left(u_{h}-u\right)\right\|^{2}-\frac{C}{\epsilon}\left\|\nabla\left(\chi-\tilde{u}_{h}\right)^{+}\right\|^{2} \\
& -C\left\|\nabla\left(u_{h}-\tilde{u}_{h}\right)\right\|^{2}-\frac{C}{\epsilon} \sum_{T \in \mathcal{T}_{h}} h_{T}^{4}\left\|\nabla \sigma_{h}\left(u_{h}\right)\right\|^{2}+C \sum_{T \in \mathbb{F}_{h} \cup \mathbb{C}_{h}} \int_{T} \sigma_{h}\left(\chi-\chi_{h}\right)^{-} d x \\
& -C \sum_{e \in \partial \mathbb{F}_{h}^{i}} \int_{e} h_{e} \llbracket \nabla\left(u_{h}-\chi_{h}\right) \rrbracket^{2} d s .
\end{aligned}
$$

Proof. Let $\hat{u}_{h}=\max \left\{\tilde{u}_{h}, \chi\right\}$. Then $\hat{u}_{h} \in \mathcal{K}$ and find

$$
\begin{aligned}
\left\langle\sigma, u-\tilde{u}_{h}\right\rangle & \left.=\left\langle\sigma,\left(u-\hat{u}_{h}\right)+\hat{u}_{h}-\tilde{u}_{h}\right)\right\rangle \\
& \geq\left\langle\sigma, \hat{u}_{h}-\tilde{u}_{h}\right\rangle,
\end{aligned}
$$

where we have used the fact that $\left\langle\sigma, u-\hat{u}_{h}\right\rangle \geq 0$, thanks to (1.3). Note by the definition of $\hat{u}_{h}$ that $\hat{u}_{h}-\tilde{u}_{h}=\left(\chi-\tilde{u}_{h}\right)^{+}$. Then

$$
\begin{aligned}
\left\langle\sigma, \hat{u}_{h}-\tilde{u}_{h}\right\rangle & =\left\langle\sigma,\left(\chi-\tilde{u}_{h}\right)^{+}\right\rangle=\left\langle\sigma-\sigma_{h},\left(\chi-\tilde{u}_{h}\right)^{+}\right\rangle+\left\langle\sigma_{h},\left(\chi-\tilde{u}_{h}\right)^{+}\right\rangle \\
& \geq-\epsilon\left\|\sigma-\sigma_{h}\right\|_{-1}^{2}-\frac{1}{\epsilon}\left\|\nabla\left(\chi-\tilde{u}_{h}\right)^{+}\right\|^{2}+\left\langle\sigma_{h},\left(\chi-\tilde{u}_{h}\right)^{+}\right\rangle,
\end{aligned}
$$

where $\epsilon>0$ is some constant which will be chosen later. Therefore

$$
\left\langle\sigma-\sigma_{h}, u-\tilde{u}_{h}\right\rangle \geq-\epsilon\left\|\sigma-\sigma_{h}\right\|_{-1}^{2}-\frac{1}{\epsilon}\left\|\nabla\left(\chi-\tilde{u}_{h}\right)^{+}\right\|^{2}+\left\langle\sigma_{h},\left(\chi-\tilde{u}_{h}\right)^{+}-\left(u-\tilde{u}_{h}\right)\right\rangle .
$$


We consider

$$
\begin{aligned}
\left\langle\sigma_{h},\left(\chi-\tilde{u}_{h}\right)^{+}-\left(u-\tilde{u}_{h}\right)\right\rangle & =\sum_{T \in \mathcal{T}_{h}} \int_{T} \sigma_{h}\left(\chi-\tilde{u}_{h}\right)^{+}-\left(u-\tilde{u}_{h}\right) d x \\
& =\sum_{T \in \mathbb{F}_{h} \cup \mathbb{C}_{h}} \int_{T} \sigma_{h}\left(\chi-\tilde{u}_{h}\right)^{+}-\left(u-\tilde{u}_{h}\right) d x
\end{aligned}
$$

since $\sigma_{h} \equiv 0$ on $\mathbb{N}_{h}$. We split the proof into a few cases.

Case 1.(Free boundary set near $\partial \Omega, \mathbb{F}_{h}$ ): Let $T \in \mathbb{F}_{h}$ and $T \cap \partial \Omega \neq \emptyset$. Then since there is at least one node $z \in \mathcal{V}_{T}$ such that $\left.\sigma_{h}\right|_{T}(z)=0$, we find by using equivalence of norms on finite dimensional spaces and scaling that

$$
\left\|\sigma_{h}\right\|_{L_{2}(T)} \leq C h_{T}\left\|\nabla \sigma_{h}\right\|_{L_{2}(T)} .
$$

If $T \cap \partial \Omega$ is an edge in $\mathcal{E}_{h}^{b}$, then since $u-\tilde{u}_{h} \in H_{0}^{1}(\Omega)$, we find by using Poincaré inequality and scaling that

$$
\left\|\tilde{u}_{h}-u\right\|_{L_{2}(T)} \leq C h_{T}\left\|\nabla\left(\tilde{u}_{h}-u\right)\right\|_{L_{2}(T)} .
$$

In the case if $T \cap \partial \Omega$ is a node in $\mathcal{V}_{h}^{b}$, we find by using the equivalence of norms on finite dimensional spaces that

$$
\left\|\Pi_{h}\left(\tilde{u}_{h}-u\right)\right\|_{L_{2}(T)} \leq C h_{T}\left\|\nabla \Pi_{h}\left(\tilde{u}_{h}-u\right)\right\|_{L_{2}(T)} \leq C h_{T}\left\|\nabla\left(\tilde{u}_{h}-u\right)\right\|_{L_{2}\left(\mathcal{T}_{T}\right)},
$$

where $\mathcal{T}_{T}:=\cup\left\{T \in \mathcal{T}_{h}: T^{\prime} \cap T \neq \emptyset\right\}$ and $\Pi_{h}: H_{0}^{1}(\Omega) \rightarrow V_{h}^{0}$ is the Clement interpolation satisfying

$$
h_{T}^{-1}\|\| \Pi_{h} v-v\left\|_{L_{2}(T)}+\right\| \nabla \Pi_{h} v\left\|_{L_{2}(T)} \leq C\right\| \nabla v \|_{L_{2}\left(\mathcal{T}_{T}\right)} \quad \forall v \in H_{0}^{1}(\Omega) .
$$

Then, we find

$$
\begin{aligned}
\left\|\tilde{u}_{h}-u\right\|_{L_{2}(T)} & \leq\left\|\left(\tilde{u}_{h}-u\right)-\Pi_{h}\left(\tilde{u}_{h}-u\right)\right\|_{L_{2}(T)}+\left\|\Pi_{h}\left(\tilde{u}_{h}-u\right)\right\|_{L_{2}(T)} \\
& \leq C h_{T}\left\|\nabla\left(\tilde{u}_{h}-u\right)\right\|_{L_{2}\left(\mathcal{T}_{T}\right)} .
\end{aligned}
$$

Therefore, we obtain

$$
\begin{aligned}
\int_{T} \sigma_{h}\left(\tilde{u}_{h}-u\right) d x \geq & -\frac{\epsilon}{2}\left\|\nabla\left(\tilde{u}_{h}-u\right)\right\|_{L_{2}\left(\mathcal{T}_{T}\right)}^{2}-\frac{C}{\epsilon} h_{T}^{4}\left\|\nabla \sigma_{h}\left(u_{h}\right)\right\|_{L_{2}(T)}^{2} \\
\geq-\epsilon\left\|\nabla\left(\tilde{u}_{h}-u_{h}\right)\right\|_{L_{2}\left(\mathcal{T}_{T}\right)}^{2}-\epsilon\left\|\nabla\left(u_{h}-u\right)\right\|_{L_{2}\left(\mathcal{T}_{T}\right)}^{2} & -C h_{T}^{4}\left\|\nabla \sigma_{h}\left(u_{h}\right)\right\|_{L_{2}(T)}^{2} .
\end{aligned}
$$

Similarly, we find that

$$
\int_{T} \sigma_{h}\left(\chi-\tilde{u}_{h}\right)^{+} d x \geq-\left\|\nabla\left(\chi-\tilde{u}_{h}\right)^{+}\right\|_{L_{2}\left(\mathcal{T}_{T}\right)}^{2}-C h_{T}^{4}\left\|\nabla \sigma_{h}\left(u_{h}\right)\right\|_{L_{2}(T)}^{2} .
$$

For the remaining proof, we note that

$$
\int_{T} \sigma_{h}\left(\left(\chi-\tilde{u}_{h}\right)^{+}-\left(u-\tilde{u}_{h}\right)\right) d x=\int_{T} \sigma_{h}\left(\left(\chi-\tilde{u}_{h}\right)^{+}-\left(\chi-\tilde{u}_{h}\right)\right) d x
$$




$$
\begin{aligned}
& +\int_{T} \sigma_{h}(\chi-u) d x \\
\geq & \int_{T} \sigma_{h}\left(\left(\chi-\tilde{u}_{h}\right)^{+}-\left(\chi-\tilde{u}_{h}\right)\right) d x,
\end{aligned}
$$

since $\sigma_{h} \leq 0$ and $\chi \leq u$. Further since any $\phi=\phi^{+}-\phi^{-}$, where $\phi^{-}=\max \{-\phi, 0\}$, we note that

$$
\int_{T} \sigma_{h}\left(\left(\chi-\tilde{u}_{h}\right)^{+}-\left(u-\tilde{u}_{h}\right)\right) d x=\int_{T} \sigma_{h}\left(\chi-\tilde{u}_{h}\right)^{-} d x .
$$

Case $2\left(\right.$ Free boundary set away from $\left.\partial \Omega, \mathbb{F}_{h}\right)$ : Let $T \in \mathbb{F}_{h}$ and $T \cap \partial \Omega=\emptyset$. Then $\tilde{u}_{h} \equiv u_{h}$ on $T$ and

$$
\begin{aligned}
\int_{T} \sigma_{h}\left(\chi-\tilde{u}_{h}\right)^{-} d x & =\int_{T} \sigma_{h}\left(\chi-u_{h}\right)^{-} d x \\
& \geq \int_{T} \sigma_{h}\left(\chi-\chi_{h}\right)^{-} d x+\int_{T} \sigma_{h}\left(u_{h}-\chi_{h}\right) d x .
\end{aligned}
$$

Since $T \in \mathbb{F}_{h}$, there is some $z \in \mathcal{V}_{T}$ such that $\left.u_{h}\right|_{T}(z)=\chi(z)$. Also since $u_{h} \geq \chi_{h}$, we find by using [44, Lemma 3.6] that

$$
\left\|u_{h}-\chi_{h}\right\|_{L_{2}(T)} \leq C h_{T}\left(\sum_{e \in \mathcal{E}_{p} \cap \mathcal{E}_{h}^{i}} \int_{e} h_{e}\left[\left[\nabla\left(u_{h}-\chi_{h}\right)\right]^{2} d s\right)^{1 / 2}\right.
$$

Also since $\left.\sigma_{h}\right|_{T}(z)=0$ for at least one $z \in \mathcal{V}_{T}$, we find using (4.6) and (4.12) that

$$
\begin{aligned}
\left|\int_{T} \sigma_{h}\left(u_{h}-\chi_{h}\right) d x\right| & \leq\left\|\sigma_{h}\right\|_{L_{2}(T)}\left\|u_{h}-\chi_{h}\right\|_{L_{2}(T)} \\
& \leq C h_{T}^{2}\left\|\nabla \sigma_{h}\right\|_{L_{2}(T)}\left(\sum_{e \in \mathcal{E}_{p} \cap \mathcal{E}_{h}^{i}} \int_{e} h_{e}\left[\nabla \nabla\left(u_{h}-\chi_{h}\right)\right]^{2} d s\right)^{1 / 2},
\end{aligned}
$$

which implies that

$$
\int_{T} \sigma_{h}\left(u_{h}-\chi_{h}\right) d x \geq-C h_{T}^{4}\left\|\nabla \sigma_{h}\right\|_{L_{2}(T)}^{2}-C \sum_{e \in \mathcal{E}_{p} \cap \mathcal{E}_{h}^{i}} \int_{e} h_{e}\left[\nabla \nabla\left(u_{h}-\chi_{h}\right)\right]^{2} d s .
$$

Case $3\left(\right.$ Contact set away from $\left.\partial \Omega, \mathbb{C}_{h}\right)$ : Let $T \in \mathbb{C}_{h}$ and $T \cap \partial \Omega=\emptyset$. Then $\tilde{u}_{h} \equiv u_{h} \equiv \chi_{h}$ on $T$ and

$$
\int_{T} \sigma_{h}\left(\chi-\tilde{u}_{h}\right)^{-} d x=\int_{T} \sigma_{h}\left(\chi-u_{h}\right)^{-} d x=\int_{T} \sigma_{h}\left(\chi-\chi_{h}\right)^{-} d x .
$$

Case $4\left(\right.$ Contact set near $\left.\partial \Omega, \mathbb{C}_{h}\right)$ : Let $T \in \mathbb{C}_{h}$ and $T \cap \partial \Omega \neq \emptyset$. Then $u_{h} \equiv \chi_{h}$ on $T$ and

$$
\begin{aligned}
\left(\chi-\tilde{u}_{h}\right)^{-} & =\max \left\{\tilde{u}_{h}-\chi, 0\right\}=\max \left\{\tilde{u}_{h}-u_{h}+\chi_{h}-\chi, 0\right\} \\
& \leq \max \left\{\tilde{u}_{h}-u_{h}, 0\right\}+\max \left\{\chi_{h}-\chi, 0\right\}
\end{aligned}
$$




$$
=\left(\tilde{u}_{h}-u_{h}\right)^{+}+\left(\chi-\chi_{h}\right)^{-} .
$$

Therefore

$$
\int_{T} \sigma_{h}\left(\chi-\tilde{u}_{h}\right)^{-} d x \geq \int_{T} \sigma_{h}\left(\tilde{u}_{h}-u_{h}\right)^{+} d x+\int_{T} \sigma_{h}\left(\chi-\chi_{h}\right)^{-} d x .
$$

Further since $\sigma_{h}(z)=0$ for $z \in \partial T \cap \partial \Omega$, we find by using equivalence of norms on finite dimensional spaces and scaling as in (4.6) that

$$
\left\|\sigma_{h}\right\|_{L_{2}(T)} \leq C h_{T}\left\|\nabla \sigma_{h}\right\|_{L_{2}(T)} .
$$

and similar to (4.7)-(4.8), we find that

$$
\left\|\left(\tilde{u}_{h}-u_{h}\right)^{+}\right\|_{L_{2}(T)} \leq\left\|\tilde{u}_{h}-u_{h}\right\|_{L_{2}(T)} \leq C h_{T}\left\|\nabla\left(\tilde{u}_{h}-u_{h}\right)\right\|_{L_{2}\left(\mathcal{T}_{T}\right)} .
$$

This completes the rest of the proof.

We combine Lemma 4.1, Lemma 4.2, Lemma 4.3 and deduce the following main result of the article.

Theorem 4.4. Let $\chi_{h} \in V_{h}$ be the Lagrange interpolation of $\chi$ and $\left.g\right|_{e} \in H^{1}(e)$ for all $e \in \mathcal{E}_{h}^{b}$. Then there holds

$$
\begin{aligned}
\left\|\nabla\left(u-u_{h}\right)\right\|^{2}+\left\|\sigma-\sigma_{h}\right\|_{-1}^{2} \leq & C\left(\eta_{f}^{2}+\eta_{J}^{2}+\eta_{\sigma}^{2}\right)+C \sum_{e \in \mathcal{E}_{h}^{b}} h_{e}\left\|\left(g-g_{h}\right)^{\prime}\right\|_{L_{2}(e)}^{2} \\
& +C\left\|\nabla\left(\chi-\tilde{u}_{h}\right)^{+}\right\|^{2}+C \sum_{T \in \mathbb{F}_{h} \cup \mathbb{C}_{h}} \int_{T}\left(-\sigma_{h}\right)\left(\chi-\chi_{h}\right)^{-} d x \\
& +C \sum_{e \in \partial \mathbb{F}_{h}^{i}} \int_{e} h_{e}\left[\nabla\left(u_{h}-\chi_{h}\right)\right]^{2} d s .
\end{aligned}
$$

Remark 4.5. The error estimator in general explicitly depends on the post-processed solution $\tilde{u}_{h}$. If $\tilde{u}_{h}$ is defined by the harmonic extension, then the error estimator is not computable. However, the linear extension can be used to construct $\tilde{u}_{h}$ and the error estimator is computable.

Under an assumption that the free boundary is interior to $\Omega$, we prove the following estimate: Note that the hypothesis we make in the corollary is easy to check.

Corollary 4.6. Suppose that for all $T \in \mathcal{T}_{h}^{b}$, there holds

$$
\max _{z \in T} \chi(z) \leq \min _{z \in \partial T} u_{h}^{*}
$$

where $u_{h}^{*}$ is defined as in (3.1). Then, we have

$$
\begin{aligned}
\left\|\nabla\left(u-u_{h}\right)\right\|^{2}+\left\|\sigma-\sigma_{h}\right\|_{-1}^{2} \leq & C\left(\eta_{f}^{2}+\eta_{J}^{2}+\eta_{\sigma}^{2}\right)+C \sum_{e \in \mathcal{E}_{h}^{b}} h_{e}\left\|\left(g-g_{h}\right)^{\prime}\right\|_{L_{2}(e)}^{2} \\
& +C \sum_{T \in \mathcal{T}_{h}^{i}}\left\|\nabla\left(\chi-u_{h}\right)^{+}\right\|_{L_{2}(T)}^{2}+C \sum_{T \in \mathbb{F}_{h} \cup \mathbb{C}_{h}} \int_{T}\left(-\sigma_{h}\right)\left(\chi-\chi_{h}\right)^{-} d x
\end{aligned}
$$




$$
+C \sum_{e \in \partial \mathbb{F}_{h}^{i}} \int_{e} h_{e}\left[\llbracket \nabla\left(u_{h}-\chi_{h}\right)\right]^{2} d s .
$$

In particular, the error estimator is independent of the post-processed solution $\tilde{u}_{h}$.

Proof. From (3.5) and (3.9), we have for any $T \in \mathcal{T}_{h}^{b}$ that

$$
\min _{z \in T} \tilde{u}_{h}(z)=\min _{z \in \partial T} u_{h}^{*}
$$

Hence by the hypothesis in the statement of the corollary, $\left(\chi-\tilde{u}_{h}\right)^{+} \equiv 0$ on any $T \in \mathcal{T}_{h}^{b}$. Further since $\tilde{u}_{h} \equiv u_{h}$ on any $T \in \mathcal{T}_{h}^{i}$, we have on any such $T$ that $\left(\chi-\tilde{u}_{h}\right)^{+} \equiv\left(\chi-u_{h}\right)^{+}$. Moreover, from (3.4) and Theorem 3.2, we have

$$
\eta_{g}^{2}=\left\|\nabla\left(u-\tilde{u}_{h}\right)\right\|^{2} \leq C \sum_{e \in \mathcal{E}_{h}^{b}} h_{e}\left\|\left(g-g_{h}\right)^{\prime}\right\|_{L_{2}(e)}^{2} .
$$

The proof now follows from Theorem 4.4,

Corollary 4.7. Suppose that $\chi \in \mathbb{P}_{1}(\Omega)$. Then

$$
\left\|\nabla\left(u-u_{h}\right)\right\|^{2}+\left\|\sigma-\sigma_{h}\right\|_{-1}^{2} \leq C\left(\eta_{f}^{2}+\eta_{J}^{2}+\eta_{\sigma}^{2}\right)+C \sum_{e \in \mathcal{E}_{h}^{b}} h_{e}\left\|\left(g-g_{h}\right)^{\prime}\right\|_{L_{2}(e)}^{2} .
$$

Proof. Since $\chi \in \mathbb{P}_{1}(\Omega)$, we have that $u_{h} \geq \chi$ on $\bar{\Omega}$. From the hypothesis on $g$, we have $g \geq \chi$ on $\partial \Omega$. These together imply that $u_{h}^{*} \geq \chi$ on $\partial T$ for any $T \in \mathcal{T}_{h}^{b}$. Further by the construction of $\tilde{u}_{h}$ (in both the choices), we have for any $T \in \mathcal{T}_{h}^{b}$ that

$$
\max _{z \in T} \tilde{u}_{h}=\max _{z \in \partial T} u_{h}^{*} \text {. }
$$

Therefore $\left(\chi-\tilde{u}_{h}\right)^{+} \equiv 0$ on $\bar{\Omega}$. This together with Theorem 4.4 completes the proof.

Remark 4.8. The post-processed solution $\tilde{u}_{h}$ defined by the linear extension is computable once the discrete solution $u_{h}$ is known. The new solution $\tilde{u}_{h}$ satisfies the exact boundary conditions. It may also be useful in other contexts.

\section{Simplified ERror Estimator}

In this section, we conclude a simplified error estimator for the obstacle problem by equivalently rewriting the model problem (1.1) as follows: Find $u \in \mathcal{K}$ such that $u=w+\chi$, where $w \in \mathcal{K}_{0}$ satisfies

$$
a(w, v-w) \geq(f, v-w)-a(\chi, v-w) \text { for all } v \in \mathcal{K}_{0}
$$

and

$$
\mathcal{K}_{0}:=\left\{v \in H^{1}(\Omega): v \geq 0 \text { a.e. in } \Omega, \gamma_{0}(v)=g-\chi \text { on } \partial \Omega\right\},
$$

It can be seen that the set $\mathcal{K}_{0}$ is nonempty as $u-\chi \in \mathcal{K}_{0}$. The discrete version of $\mathcal{K}_{0}$ can be defined as

$$
\mathcal{K}_{0 h}:=\left\{v \in V_{h}: v \geq 0 \text { a.e. in } \Omega, \gamma_{0}(v)=g_{h}-\chi_{h} \text { on } \partial \Omega\right\},
$$


where $\chi_{h} \in V_{h}$ is the Lagrange interpolation of $\chi$. The discrete problem (2.2) also can be equivalently rewritten as to find $u_{h} \in \mathcal{K}_{h}$ such that $u_{h}=w_{h}+\chi_{h}$, where $w_{h} \in \mathcal{K}_{0 h}$ satisfies

$$
a(w, v-w) \geq(f, v-w)-a\left(\chi_{h}, v-w\right) \text { for all } v \in \mathcal{K}_{0 h} .
$$

Let $\gamma_{0}(\chi) \in H^{1}(e)$ for all $e \in \mathcal{E}_{h}^{b}$. Then the Dirichlet data $g-\chi$ satisfies the hypothesis of Theorem 4.4 and hence the Corollary 4.7 deduce the following result under the assumption that $\gamma_{0}(\chi) \in H^{1}(e)$ for all $e \in \mathcal{E}_{h}^{b}$ :

Theorem 5.1. Let $u \in \mathcal{K}\left(\right.$ resp. $\left.u_{h} \in \mathcal{K}_{h}\right)$ be the solution of (1.1) (resp. (2.2)). Assume that the obstacle $\chi$ and the Dirichlet data $g$ satisfy $\gamma_{0}(\chi) \in H^{1}(e)$ and $g \in H^{1}(e)$ for all $e \in \mathcal{E}_{h}^{b}$. Then there holds

$$
\begin{aligned}
\left\|\nabla\left(u-u_{h}\right)\right\|^{2}+\left\|\sigma-\sigma_{h}\right\|_{-1}^{2} \leq & C\left(\eta_{f}^{2}+\eta_{J}^{2}+\eta_{\sigma}^{2}\right)+C\left\|\nabla\left(\chi-\chi_{h}\right)\right\|^{2} \\
& +C \sum_{e \in \mathcal{E}_{h}^{b}} h_{e}\left\|\left(g-g_{h}\right)^{\prime}\right\|_{L_{2}(e)}^{2}+C \sum_{e \in \mathcal{E}_{h}^{b}} h_{e}\left\|\left(\chi-\chi_{h}\right)^{\prime}\right\|_{L_{2}(e)}^{2} .
\end{aligned}
$$

Note that the error estimator in Theorem 5.1 does not involve any min/max functions (which are non smooth and not easy to compute in the implementation).

5.1. Local Efficiency Estimates: The terms

$$
\left(\sum_{e \in \mathcal{E}_{h}^{b}} h_{e}\left\|\left(g-g_{h}\right)^{\prime}\right\|_{L_{2}(e)}^{2}\right)^{1 / 2}, \quad\left(\sum_{e \in \mathcal{E}_{h}^{b}} h_{e}\left\|\left(\chi-\chi_{h}\right)^{\prime}\right\|_{L_{2}(e)}^{2}\right)^{1 / 2} \text { and }\left\|\nabla\left(\chi-\chi_{h}\right)\right\|
$$

are considered to be the data approximations. We will prove the local efficiency of the remaining error estimators.

Theorem 5.2. There holds

$$
\begin{aligned}
h_{T}\left\|f-\sigma_{h}\right\|_{L_{2}(T)} & \leq C\left(\left|u-u_{h}\right|_{H^{1}(T)}+\left\|\sigma-\sigma_{h}\right\|_{H^{-1}(T)}+h_{T}\|f-\bar{f}\|_{L_{2}(T)}\right), \\
h_{T}^{2}\left\|\nabla \sigma_{h}\right\|_{L_{2}(T)} & \leq C\left(\left|u-u_{h}\right|_{H^{1}(T)}+\left\|\sigma-\sigma_{h}\right\|_{H^{-1}(T)}+h_{T}\|f-\bar{f}\|_{L_{2}(T)}\right),
\end{aligned}
$$

for any $T \in \mathcal{T}_{h}$ and $\bar{f} \in \mathbb{P}_{0}(T)$. Furthermore for any $e \in \mathcal{E}_{h}^{i}$, there holds

$$
h_{e}^{1 / 2}\left\|\llbracket \nabla u_{h} \rrbracket\right\|_{L_{2}(e)} \leq C\left(\left|u-u_{h}\right|_{H^{1}\left(\mathcal{T}_{e}\right)}+\left\|\sigma-\sigma_{h}\right\|_{H^{-1}\left(\mathcal{T}_{e}\right)}+h_{e}\|f-\bar{f}\|_{L_{2}\left(\mathcal{T}_{e}\right)}\right),
$$

where $\mathcal{T}_{e}$ is the patch of two triangles $T_{ \pm}$sharing the edge e, see Fig [5.1.

Proof. The proof follows by bubble function techniques [45, 46] [44. Let $\left.\bar{f}\right|_{T} \in \mathbb{P}_{0}(T)$ for all $T \in \mathcal{T}_{h}$. Let $T \in \mathcal{T}_{h}$ be arbitrary and $b_{T} \in \mathbb{P}_{3}(T) \cap H_{0}^{1}(T)$ be the bubble function defined on $T$ such that $b_{T}\left(x_{T}\right)=1$, where $x_{T}$ is the barycenter of $T$. Let $\phi=b_{T}\left(\bar{f}-\sigma_{h}\right)$ on $T$ and extend it to be zero on $\Omega \backslash T$. We have for some mesh independent constants $C_{1}$ and $C_{2}$ that

$$
C_{1}\left\|\bar{f}-\sigma_{h}\right\|_{L_{2}(T)} \leq\|\phi\|_{L_{2}(T)} \leq C_{2}\left\|\bar{f}-\sigma_{h}\right\|_{L_{2}(T)} .
$$

It follows from (1.2) and integration by parts that

$$
\int_{T}\left(f-\sigma_{h}\right) \phi d x=\int_{T}\left(f-\sigma_{h}\right) \phi d x+\int_{T} \Delta u_{h} \phi d x
$$




$$
\begin{aligned}
& =\int_{\Omega} f \phi d x-\int_{\Omega} \nabla u_{h} \cdot \nabla \phi d x-\left\langle\sigma_{h}, \phi\right\rangle \\
& =\int_{\Omega} \nabla\left(u-u_{h}\right) \cdot \nabla \phi d x+\left\langle\sigma-\sigma_{h}, \phi\right\rangle \\
& =\int_{T} \nabla\left(u-u_{h}\right) \cdot \nabla \phi d x+\left\langle\sigma-\sigma_{h}, \phi\right\rangle_{H^{-1}(T) \times H_{0}^{1}(T)}
\end{aligned}
$$

Using a standard inverse estimate [9, 17], we find

$$
\begin{aligned}
C_{1}\left\|\bar{f}-\sigma_{h}\right\|_{L_{2}(T)}^{2} & \leq \int_{T}\left(\bar{f}-\sigma_{h}\right) \phi d x=\int_{T}(\bar{f}-f) \phi d x+\int_{T}\left(f-\sigma_{h}\right) \phi d x \\
& =\int_{T}(\bar{f}-f) \phi d x+\int_{T} \nabla\left(u-u_{h}\right) \cdot \nabla \phi d x+\left\langle\sigma-\sigma_{h}, \phi\right\rangle_{H^{-1}(T) \times H_{0}^{1}(T)} \\
& \leq\|f-\bar{f}\|_{L_{2}(T)}\|\phi\|_{L_{2}(T)}+\left(\left|u-u_{h}\right|_{H^{1}(T)}+\left\|\sigma-\sigma_{h}\right\|_{H^{-1}(T)}\right)|\phi|_{H^{1}(T)} \\
& \leq\left(\|f-\bar{f}\|_{L_{2}(T)}+C h_{T}^{-1}\left(\left|u-u_{h}\right|_{H^{1}(T)}+\left\|\sigma-\sigma_{h}\right\|_{H^{-1}(T)}\right)\right)\|\phi\|_{L_{2}(T)} \\
& \leq C\left(\|f-\bar{f}\|_{L_{2}(T)}+h_{T}^{-1}\left(\left|u-u_{h}\right|_{H^{1}(T)}+\left\|\sigma-\sigma_{h}\right\|_{H^{-1}(T)}\right)\right)\left\|\bar{f}-\sigma_{h}\right\|_{L_{2}(T)},
\end{aligned}
$$

which implies

$$
h_{T}\left\|\bar{f}-\sigma_{h}\right\|_{L_{2}(T)} \leq C\left(\left|u-u_{h}\right|_{H^{1}(T)}+\left\|\sigma-\sigma_{h}\right\|_{H^{-1}(T)}+h_{T}\|f-\bar{f}\|_{L_{2}(T)}\right) .
$$

Using the triangle inequality, we obtain

$$
h_{T}\left\|f-\sigma_{h}\right\|_{L_{2}(T)} \leq C\left(\left|u-u_{h}\right|_{H^{1}(T)}+\left\|\sigma-\sigma_{h}\right\|_{H^{-1}(T)}+h_{T}\|f-\bar{f}\|_{L_{2}(T)}\right) .
$$

This completes the proof of (5.3). Using an inverse inequality [9, 17], note that

$$
h_{T}^{2}\left\|\nabla \sigma_{h}\right\|_{L_{2}(T)}=h_{T}^{2}\left\|\nabla\left(\bar{f}-\sigma_{h}\right)\right\|_{L_{2}(T)} \leq C h_{T}\left\|\bar{f}-\sigma_{h}\right\|_{L_{2}(T)} .
$$

An appeal to (5.6), completes the proof of (5.4). We next prove (5.5) using similar bubble function techniques. Let $e \in \mathcal{E}_{h}^{i}$ and $T_{ \pm}$be the triangles sharing this edge $e$. Denote by $\mathcal{T}_{e}$ the patch of the two triangles $T_{ \pm}$(cf. Fig. 5.1).

Now consider $\left[\nabla u_{h} \rrbracket\right.$ on $e$ and extend it to $T_{ \pm}$so that it is constant along the lines orthogonal to $e$. Denote the resulting function by $\zeta_{1} \in \mathbb{P}_{0}\left(\mathcal{T}_{e}\right)$. It is then obvious that $\zeta_{1}=\llbracket \nabla u_{h} \rrbracket$ on $e$. Construct a piecewise polynomial bubble function $\zeta_{2} \in H_{0}^{1}\left(\mathcal{T}_{e}\right)$ such that $\zeta_{2}\left(x_{e}\right)=1$, where $x_{e}$ is the midpoint of $e$. Denote $\phi=\zeta_{1} \zeta_{2}$ and extend $\phi$ to be zero on $\Omega \backslash \mathcal{T}_{e}$. We have by scaling [45],

$$
C\|\phi\|_{L_{2}\left(\mathcal{T}_{e}\right)} \leq\left(\int_{e} h_{e}\left[\nabla \nabla u_{h}\right]^{2} d s\right)^{1 / 2},
$$

for some mesh independent constant $C$. Then, using (1.2), Poincaré's inequality and a standard inverse inequality [9, 17], we find

$$
\int_{e} \llbracket\left[\nabla u_{h} \rrbracket^{2} d s \leq C \int_{e} \llbracket \nabla u_{h} \rrbracket \zeta_{1} \zeta_{2} d s=C \int_{\mathcal{T}_{e}} \nabla u_{h} \cdot \nabla \phi d x\right.
$$




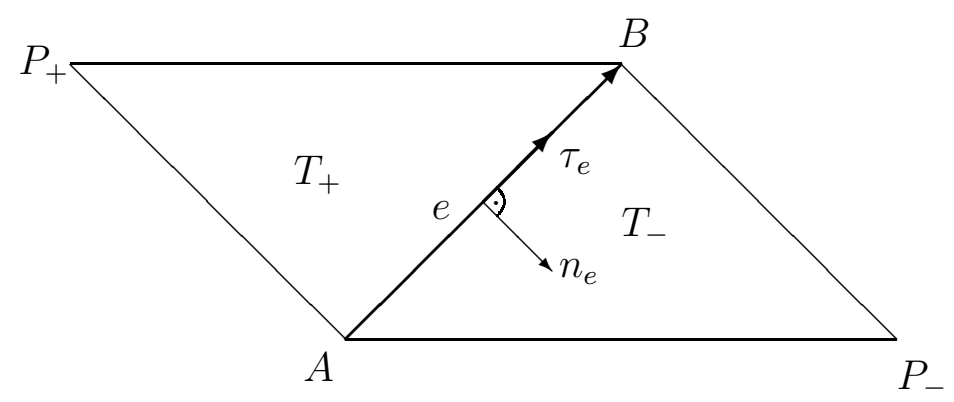

Figure 5.1. Two neighboring triangles $T_{+}$and $T_{1}$ that share the edge $e=$ $\partial T_{+} \cap \partial T_{-}$with initial node $A$ and end node $B$ and unit normal $n_{e}$. The orientation of $n_{e}=n_{+}=-n_{-}$equals the outer normal of $T_{+}$, and hence, points into $T_{-}$.

$$
\begin{aligned}
& =C\left(\int_{\mathcal{T}_{e}} \nabla\left(u_{h}-u\right) \cdot \nabla \phi d x+\int_{\mathcal{T}_{e}} f \phi d x-\langle\sigma, \phi\rangle\right) \\
& =C\left(\int_{\mathcal{T}_{e}} \nabla\left(u_{h}-u\right) \cdot \nabla \phi d x+\int_{\mathcal{T}_{e}}\left(f-\sigma_{h}\right) \phi d x-\left\langle\sigma-\sigma_{h}, \phi\right\rangle\right) \\
& \leq C\left(h_{e}\left\|f-\sigma_{h}\right\|_{L_{2}\left(\mathcal{T}_{e}\right)}+\left|u-u_{h}\right|_{H^{1}\left(\mathcal{T}_{e}\right)}+\left\|\sigma-\sigma_{h}\right\|_{H^{-1}\left(\mathcal{T}_{e}\right)}\right)|\phi|_{H^{1}\left(\mathcal{T}_{e}\right)} \\
& \leq C\left(h_{e}\left\|f-\sigma_{h}\right\|_{L_{2}\left(\mathcal{T}_{e}\right)}+\left|u-u_{h}\right|_{H^{1}\left(\mathcal{T}_{e}\right)}+\left\|\sigma-\sigma_{h}\right\|_{H^{-1}\left(\mathcal{T}_{e}\right)}\right) h_{e}^{-1}\|\phi\|_{L^{2}\left(\mathcal{T}_{e}\right)} .
\end{aligned}
$$

Finally using (5.7), (5.4) and (15.3), we complete the proof of (5.5).

\section{NumericAl EXPERIMENTS}

In this section, we present some numerical experiments. Let $\Omega$ be the unit disk and the obstacle function to be $\chi=1-2 r^{2}$, where $r=\sqrt{x^{2}+y^{2}}$. The load function $f$ is taken to be

$$
f(r):= \begin{cases}0 & \text { if } r<r_{0}, \\ 4 r_{0} / r & \text { if } r \geq r_{0},\end{cases}
$$

in such a way that the solution $u$ takes the form

$$
u(r):= \begin{cases}1-2 r^{2} & \text { if } r<r_{0}, \\ 4 r_{0}(1-r) & \text { if } r \geq r_{0},\end{cases}
$$

where $r_{0}=(\sqrt{2}-1) / \sqrt{2}$.

In the experiment, we use the adaptive algorithm consisting of four successive modules

$$
\mathrm{SOLVE} \rightarrow \mathrm{ESTIMATE} \rightarrow \mathrm{MARK} \rightarrow \mathrm{REFINE}
$$




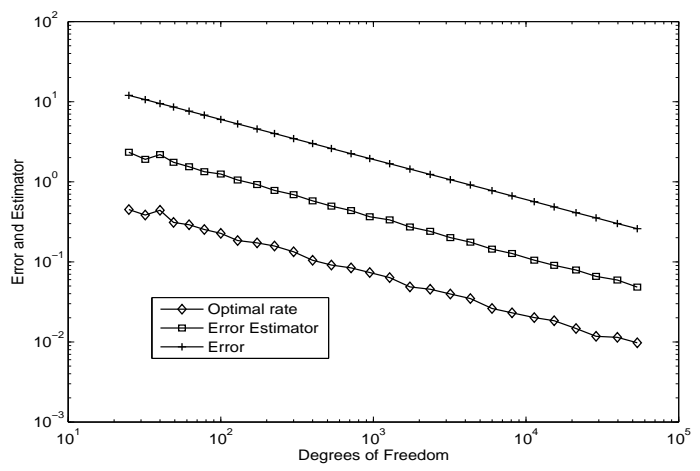

FiguRE 6.1. Error and Estimator

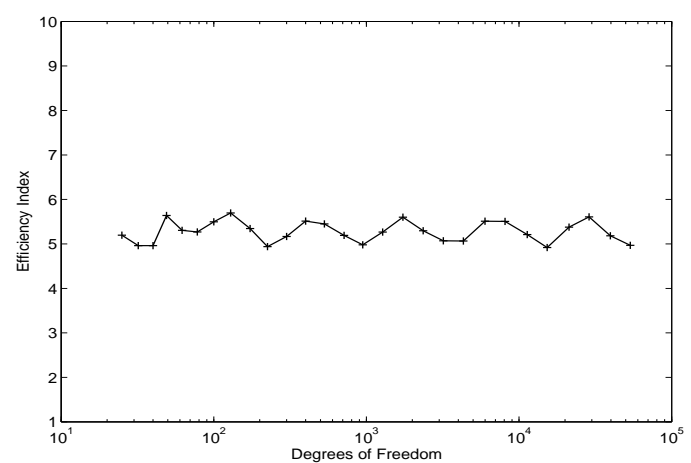

Figure 6.2. Efficiency Index

We use the primal-dual active set strategy [31] in the step SOLVE to solve the discrete obstacle problem. The estimator derived in Theorem 5.1 is computed in the step ESTIMATE and then the Dörfler's bulk marking strategy [18] with parameter $\theta=0.3$ has been used in the step MARK to mark the elements for refinement. Using the newest vertex bisection algorithm, we refine the mesh and obtain a new mesh. The convergence history of errors and estimators is depicted in Figure 6.1. The figure illustrates the optimal order of convergence as well as the reliability of the error estimator. The efficiency index := estimator/error is computed and plotted in Figure 6.2. The free boundary set and the contact set have been captured by the error estimator efficiently see Figure 6.3. The experiment clearly illustrates the theoretical results derived in the article.

\section{Conclusions}

The a posteriori error analysis of finite element method for the elliptic obstacle problem is revisited. By rewriting general obstacle problem into a problem with zero obstacle and inhomogeneous Dirichlet boundary condition, we have derived reliable and efficient error 


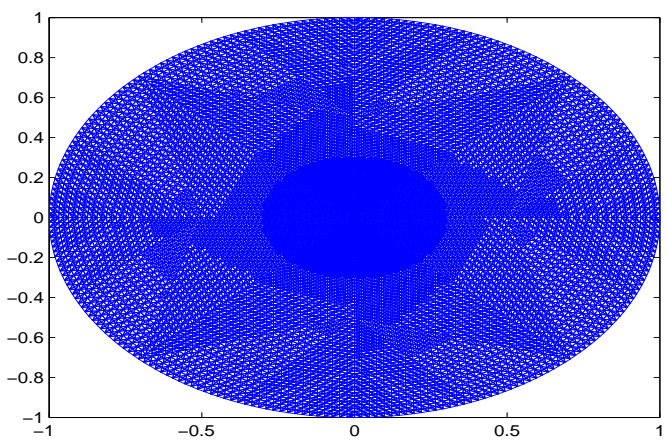

Figure 6.3. Mesh Refinement at Intermediate Level

bounds that are simpler and easily computable. The analysis covers the inhomogeneous Dirichelt boundary condition by constructing suitable post-processed solutions that satisfy the boundary conditions exactly. We have proposed two post-processing methods in which one is by harmonic extension near the boundary and the other one is by linear interpolation. These constructions are possible in three dimensions with appropriate modifications and further the harmonic extension is directly extendable. Numerical experiments illustrate the theoretical results.

\section{REFERENCES}

[1] M. Ainsworth and J. T. Oden. A posteriori error estimation in finite element analysis. Pure and Applied Mathematics (New York). Wiley-Interscience [John Wiley \& Sons], New York, 2000.

[2] M. Ainsworth, J. T. oden and C. Y. Lee. Local a posteriori error estimates for variational inequalities. Numer. Meth. PDEs, 9:23-33, 1993.

[3] K. Atkinson and W. Han. Theoretical Numerical Analysis. A functional analysis framework. Thrid edition, Springer, 2009.

[4] L. Banz and E. P. Stephan. A posteriori error estimates of $h p$-adaptive IPDG-FEM for elliptic obstacle problems. Appl. Numer. Math., 76:76-92, 2014.

[5] S. Bartels and C. Carstensen. Averaging techniques yield relaible a posteriori finite element error control for obstacle problems, Numer. Math.. 99:225-249, 2004.

[6] F. Ben Belgacem. Numerical simulation of some variational inequalities arisen from unilateral contact problems by the finite element method. SIAM J. Numer. Anal., 37:11981216, 2000.

[7] H. Blum and F. T. Suttmeier. An adaptive finite element discretization for a simplified Signorini problem. Calcolo, 37:65-77, 2000.

[8] D. Braess. A posteriori error estimators for obstacle problems-another look. Numer. Math., 101:415-421, 2005.

[9] S.C. Brenner and L.R. Scott. The Mathematical Theory of Finite Element Methods (Third Edition). Springer-Verlag, New York, 2008.

[10] S. C. Brenner, L. Sung and Y. Zhang. Finite element methods for the displacement obstacle problem of clamped plates. Math. Comp., 81: 1247-1262, 2012. 
[11] S. C. Brenner, L. Sung, H. Zhang and Y. Zhang. A quadratic C 0 interior penalty method for the displacement obstacle problem of clamped Kirchhoff plates. SIAM J. Numer. Anal., 50: 3329-3350, 2012.

[12] F. Brezzi, W. W. Hager, and P. A. Raviart. Error estimates for the finite element solution of variational inequalities, Part I. Primal theory. Numer. Math., 28:431-443, 1977.

[13] D. Braess, C. Carstensen and R.H.W. Hoppe. Convergence analysis of a conforming adaptive finite element method for an obstacle problem. Numer. Math., 107:455-471, 2007.

[14] D. Braess, C. Carstensen and R. Hoppe. Error reduction in adaptive finite element approximations of elliptic obstacle problems. J. Comput. Math., 27:148-169, 2009.

[15] C. Carstensen. Efficiency of a posteriori BEM-error estimates for first-kind integral equations on quasiuniform meshes. Math. Comp., 65:69-84, 1996.

[16] Z. Chen and R. Nochetto. Residual type a posteriori error estimates for elliptic obstacle problems. Numer. Math., 84:527-548, 2000.

[17] P.G. Ciarlet. The Finite Element Method for Elliptic Problems. North-Holland, Amsterdam, 1978.

[18] W. Dörlfer. A convergent adaptive algorithm for Poisson's equation. SIAM J. Numer. Anal., 33:11061124, 1996.

[19] G. Drouet and P. Hild. Optimal convergence for discrete variational inequalities modelling signorini contact in $2 \mathrm{~d}$ and $3 \mathrm{~d}$ without additional assumptions on the unknown contact set. SIAM J. Numer. Anal., 53:1488-1507, 2015.

[20] R. S. Falk. Error estimates for the approximation of a class of variational inequalities. Math. Comp., 28:963-971, (1974).

[21] M. Feischl, M. Page and D. Praetorius. Convergence of adaptive FEM for some elliptic obstacle problem with inhomogeneous Dirichlet data. Int. J. Numer. Anal. Model., 11:229-253, 2014.

[22] R. Glowinski. Numerical Methods for Nonlinear Variational Problems. Springer-Verlag, Berlin, 2008.

[23] Q. Zou, A. Veeser, R. Kornhuber and C. Gräser. Hierarchical error estimates for the energy functional in obstacle problems. Numer. Math., 117:653-677, 2011.

[24] T. Gudi and K. Porwal. A posteriori error control of discontinuous Galerkin methods for elliptic obstacle problems. Math. Comput., 83:579-602, 2014.

[25] T. Gudi and K. Porwal. A remark on the a posteriori error analysis of discontinuous Galerkin methods for obstacle problem. Comput. Meth. Appl. Math., 14:71-87, 2014.

[26] T. Gudi and K. Porwal. A reliable residual based a posteriori error estimator for a quadratic finite element method for the elliptic obstacle problem. Comput. Methods Appl. Math., 15:145-160, 2015.

[27] J. Gwinner. On the $p$-version approximation in the boundary element method for a variational inequality of the second kind modelling unilateral contact and given friction. Appl. Numer. Math., 59:2774-2784, 2009.

[28] J. Gwinner. $h p$ - FEM convergence for unilateral contact problems with Tresca friction in plane linear elastostatics. J. Comput. Appl. Math., 254:175-184, 2013.

[29] P. Hild and Y. Renard. An improved a priori error analysis for finite element approximations of signorinis problem. SIAM J. Numer. Anal., 50:2400-2419, 2012.

[30] P. Hild and S. Nicaise. A posteriori error estimates of residual type for Signorini's problem. Numer. Math, 101:523-549, 2005.

[31] M. Hintermüller, K. Ito and K. Kunish. The primal-dual active set strategy as a semismooth Newton method. SIAM J. Optim., 13:865-888, 2003.

[32] R. H. W. Hoppe and R. Kornhuber. Adaptive multilevel methods for obstacle problems. SIAM J. Numer. Anal., 31:301-323, 1994.

[33] S. Hüeber and B. I. Wohlmuth. An optimal a priori error estimate for nonlinear multibody contact problems. SIAM J. Numer. Anal., 43:156-173, 2005. 
[34] S. Kesavan. Topics in Functional Analysis and Applications. New Age International Ltd, Publishers, New Delhi, 1989.

[35] D. Kinderlehrer and G. Stampacchia. An Introduction to Variational Inequalities and Their Applications. SIAM, Philadelphia, 2000.

[36] H. L. Royden. Real Analysis. Third edition. Prnetice-Hall of India Pvt. Ltd, New Delhi 2008.

[37] R. Nochetto, K. Siebert, and A. Veeser. Fully localized a posteriori error estimators and barrier sets for contact problems. SIAM J. Numer. Anal., 42:2118-2135, 2005.

[38] R. Nochetto, K. Siebert, and A. Veeser. Pointwise a posteriori error control for elliptic obstacle problems. Numer. Math., 95:163-195, 2003.

[39] R. Nochetto, T. V. Petersdorff and C. S. Zhang. A posteriori error analysis for a class of integral equations and variational inequalities. Numer. Math, 116:519-552, 2010.

[40] M. Page and D. Praetorius. Convergence of adaptive FEM for some elliptic obstacle problem. Appl. Anal., 92:595-615, 2013.

[41] A. Schröder. Mixed finite element methods of higher-order for model contact problems, SIAM J. Numer. Anal., 49:2323-2339, 2011.

[42] K. Siebert and A. Veeser. A uniliterally constrained quadratic minimization with adaptive finite elements. SIAM. J. Optim., 18:260-289, 2007.

[43] F. T. Suttmeier. Numerical solution of Variational Inequalities by Adaptive Finite Elements. Vieweg+Teubner Research, 2008.

[44] A. Veeser. Efficient and Relaible a posteriori error estimators for elliptic obstacle problems. SIAM J. Numer. Anal., 39:146-167, 2001.

[45] R. Verfürth. A posteriori error estimation and adaptive mesh-refinement techniques. In Proceedings of the Fifth International Congress on Computational and Applied Mathematics (Leuven, 1992), volume 50, pages 67-83, 1994.

[46] R. Verfürth. A Review of A Posteriori Error Estmation and Adaptive Mesh-Refinement Techniques. Wiley-Teubner, Chichester, 1995.

[47] L. Wang. On the quadratic finite element approximation to the obstacle problem. Numer. Math., 92:771$778,2002$.

[48] F. Wang, W. Han and X.Cheng. Discontinuous Galerkin methods for solving elliptic variational inequalities. SIAM J. Numer. Anal., 48:708-733, 2010.

[49] F. Wang, W. Han, and J. Eichholz and X. Cheng. A posteriori error estimates for discontinuous Galerkin methods of obstacle problems. Nonlinear Anal. Real World Appl., 22:664-679, 2015.

[50] A. Weiss and B. I. Wohlmuth. A posteriori error estimator for obstacle problems. SIAM J. Numer. Anal., 32:2627-2658, 2010.

[51] A. Wiess and B. I. Wohlmuth. A posteriori error estimator and error control for contact problems. Math. Comp., 78:1237-1267, 2009.

Department of Mathematics, Indian Institute of Science, Bangalore - 560012

E-mail address: sharat12@math.iisc.ernet.in

Department of Mathematics, Indian Institute of Science, Bangalore - 560012

E-mail address: gudi@math.iisc.ernet.in 